\title{
A PROVISIONAL SOLUTION TO THE NORMAL MOORE SPACE PROBLEM
}

\author{
PETER J. NYIKOS
}

\begin{abstract}
The Product Measure Extension Axiom (PMEA), whose consistency would follow from the existence of a strongly compact cardinal, implies that every normalized collection of sets in a space of character less than the continuum is well separated. Consistency of PMEA would thus solve many well-known problems of general topology, including that of whether every first countable normal space is collectionwise normal, as well as the normal Moore space problem.
\end{abstract}

1. Introduction. Ever since it was raised by F. Burton Jones in 1933 [14], the problem of whether every normal Moore space is metrizable (often referred to simply as "the normal Moore space problem") has been one of the most famous and intensively researched problems in general topology. A few of the milestones in the vast literature on this problem are: [14] Jones's own article, which includes the proof that the axiom $2^{N_{0}}<2^{N_{1}}$ [now known to be independent of the usual (Zermelo-Fraenkel with Choice) axioms of set theory] implies that every separable normal Moore space is metrizable; [3] R. H. Bing's 1951 paper, which includes a proof that every collectionwise normal Moore space is metrizable and the first example of a normal space which is not collectionwise normal; [1], [2] the papers of P. S. Alexandroff and A. V. Arhangel'skii giving various characterizations of metacompact Moore spaces (see \$2) and raising the question of whether the normal ones are metrizable; [12] R. W. Heath's proof that the existence of a separable nonmetrizable normal Moore space is equivalent to the existence of a $Q$-set of real numbers, from which also follows the existence of a metacompact nonmetrizable normal Moore space; [16], [22] the Silver-Rothberger proof that the existence of $Q$-set is consistent with (and therefore independent of) the usual axioms of set theory; and [9], [10] W. Fleissner's proof that Gödel's Axiom of Constructibility implies every normal Moore space-indeed, every normal space of character $<\mathfrak{c}-$ is collectionwise Hausdorff. A more detailed survey of the history of the problem may be found in the articles of Mary Ellen Rudin [15], [16] and Franklin Tall [22].

Work on the normal Moore space problem has led to other questions which will probably be of greater interest to a general audience than the original problem. One is whether every first countable normal space is collectionwise normal. Since Moore spaces are first countable, an affirmative solution to this problem would

Received by the editors October 25, 1978 and, in revised form, January 11, 1979.

AMS (MOS) subject classifications (1970). Primary 54D15, 54E35; Secondary 02K35, 54E30, 54A25.

Key words and phrases. Moore space, collectionwise normal, normalized, well separated, Product Measure Extension Axiom, metrizable, strongly compact. 
imply an affirmative solution to the normal Moore space problem, because of Bing's theorem. Of course, Silver's result gives us models of set theory where the solution to both problems is negative.

More generally, there is the problem [21] of whether every normalized collection of sets in a first countable space is well separated. "Normalized" and "well separated" (see definitions below) are such rudimentary concepts that this problem belongs as much to set theory as it does to topology.

In this paper, I will show that the Product Measure Extension Axiom (PMEA), defined below, gives an affirmative answer to all these problems, even if "of character $<c$ " is substituted for "first countable." Thus, if PMEA is consistent, then the existence of a nonmetrizable normal Moore space, etc. is actually independent of the usual axioms of set theory. Unfortunately, the consistency of PMEA implies that consistency of there being a measurable cardinal. Therefore (cf. [7]) we cannot hope to prove the consistency of PMEA within the usual set theory (ZFC). It may even turn out that there are no measurable cardinals in any model of set theory. However, as Kunen has shown, the consistency of PMEA would follow from the existence of a strongly compact cardinal, whose consistency has been conjectured by many set theorists. So we may regard the normal Moore space problem as provisionally solved, always continuing the search for a more "secure" model of set theory in which these consequences of PMEA hold. One could also take the opposite approach, attempting to find a "real" example of a first countable normal space which is not collectionwise normal. This would prove that there cannot be any strongly compact cardinals, and perhaps even that there are no measurable cardinals! A further discussion of the status of PMEA appears in \$3, below.

2. The main results. For the definition of a Moore space, cf. [8], [15], or [20]. We will not need it in this paper, only Bing's result that collectionwise normal Moore spaces are metrizable.

Definition 1. A collection of (disjoint) subsets $\left\{C_{\gamma} \mid \gamma \in \Gamma\right\}$ of a topological space $X$ is well separated if there is a collection of disjoint open subsets $\left\{U_{\gamma} \mid \gamma \in \Gamma\right\}$ of $X$ such that $C_{\gamma} \subset U_{\gamma}$ for all $\gamma$. It is normalized if for every subset $\Lambda$ of $\Gamma$ there are disjoint open subsets $U$ and $V$ containing $U\left\{C_{\gamma} \mid \gamma \in \Lambda\right\}$ and $U\left\{C_{\gamma} \mid \gamma \notin \Lambda\right\}$ respectively. It is discrete if each point of $X$ has a neighborhood meeting at most one of the sets $C_{\gamma}$.

It is easy to see that every well separated collection is normalized, as is every discrete collection of sets in a normal space. (In fact, this characterizes normal spaces.)

Definition 2. A space is collectionwise normal if every discrete collection of closed sets is well separated. [One gets an equivalent definition by dropping "closed."]

Definition 3. A space is first countable if every point has a countable base of neighborhoods. If $\kappa$ is a cardinal number, a space is of character $<\kappa$ if every point has a base of cardinal $<\kappa$ for its neighborhoods.

The PMEA axiom has to do with the usual measure on the product of two-point 
sets. If one take the sets to be groups with the discrete topology, it is the usual Haar measure [11]. An easy description is by way of basic closed-and-open sets. Let the product be the set of all functions from a fixed set $A$ into $\{0,1\}$. Every basic open set $U$ is the set of all functions which take on a specified value at each element of a specified finite subset $F$ of $A$. The measure of $U$ is then $1 / 2^{n}$ where $n=|F|$.

Defintion 4. Let $\mathrm{c}$ be the cardinality of the continuum. A measure $\mu$ on a set $X$ is $c$-additive if whenever $\mathbb{Q}$ is a collection of fewer than $c$ disjoint subsets of $X, \mu(\cup \mathbb{Q})=\Sigma_{A \in \mathbb{Q}} \mu(A)$. In particular, all but countably many members of $\mathbb{Q}$ are of measure 0 .

The Product Measure Extension Axiom (PMEA) is that the usual product measure on $2^{\lambda}$ (where $\lambda$ is any cardinal number) can be extended to a c-additive measure $\mu$ defined on all subsets of $2^{\lambda}$.

The proof of the first and most important theorem uses the huge variety of pairs of disjoint open sets which "normalized" gives us. The underlying idea is to pick a neighborhood of each point which is contained in some member of "enough" (in the sense of measure) pairs, and hence disjoint from the other member. The measure makes it possible to insure that if $p \in C_{\alpha}, q \in C_{\beta}, \alpha \neq \beta$, then the neighborhoods we choose for $p$ and $q$ will have an associated pair in common such that the neighborhood of $p$ is in one member of the pair, that of $q$ is in the other.

THEOREM 1 [PMEA]. Let $X$ be a space of character < c. Every normalized collection of subsets of $X$ is well separated.

Proof. Let $X$ be a space of character $<c$ and let $\left\{C_{\alpha} \mid \alpha<\lambda\right\}$ be a normalized family of subsets of $X$. Thus for each $A \subset \lambda$, there exist disjoint open subsets $U_{A}$ and $V_{A}$ of $X$ containing $\cup\left\{C_{\alpha} \mid \alpha \in A\right\}$ and $\cup\left\{C_{\alpha} \mid \alpha \notin A\right\}$ respectively.

Let $\mu$ be a c-additive measure, extending the usual product measure on $2^{\lambda}$, such that $\mu(\mathbb{Q})$ exists for all $\mathbb{Q} \subset 2^{\lambda}$. We identify $\lambda$ with the set of all ordinals whose cardinal is $<\lambda$. For each $\alpha \in \lambda$, let $\mathscr{B}_{\alpha}=\left\{f \in 2^{\lambda} \mid f(\alpha)=1\right\}$. Note that $\mu\left(2^{\lambda}\right)=1$ and $\mu\left(\mathscr{B}_{\alpha}-\mathscr{B}_{\beta}\right)=1 / 4$ for all $\alpha, \beta \in \lambda, \alpha \neq \beta$.

Each $f \in 2^{\lambda}$ is the characteristic function of some subset $A_{f}$ of $\lambda: A_{f}=\{\alpha \in$ $\lambda \mid f(\alpha)=1\}$.

For a given $\alpha$ and a given $p \in C_{\alpha}$, let $\left\{U_{\gamma}(p) \mid \gamma<\kappa_{p}\right\}$ be a base of open sets for the neighborhoods of $p$, with $\kappa_{p}<c$. Let $\mathbb{Q}[p, \gamma]=\left\{f \in 2^{\lambda} \mid U_{\gamma}(p) \subset U_{A_{f}}\right.$ or $U_{\gamma}(p)$ $\left.\subset V_{A_{A}}\right\}$. For a fixed $p, \cup\left\{\mathfrak{Q}[p, \gamma] \mid \gamma<\kappa_{p}\right\}=2^{\lambda}$. This is because, for any given $f \in 2^{\lambda}$, either $U_{A_{f}}$ or $V_{A_{f}}$ is an open set containing $C_{\alpha}$, hence there is a $\gamma$ such that $U_{\gamma}(p)$ is contained in the one which contains $C_{\alpha}$.

Because $\mu$ is c-additive and $\left\{\mathfrak{Q}[p, \gamma] \mid \gamma<\kappa_{p}\right\}$ is a collection of fewer than $c$ subsets that fill up $2^{\lambda}$, the transfinite sequence $\left\{\mu\left(\cup_{\gamma<\delta} \mathbb{Q}[p, \gamma]\right) \mid 0<\delta<\kappa_{p}\right\}$ converges monotonically to 1 . By the principle of Archimedean order, there exists a finite set of indices $\left\{\gamma_{1}(p), \ldots, \gamma_{\eta_{p}}(p)\right\}$ such that $\mu\left(\cup_{i=1}^{\eta_{1}} \mathscr{Q}\left[p, \gamma_{i}(p)\right]\right)>7 / 8$. Let $\gamma_{p}$ be such that $U_{\gamma_{p}}(p) \subset \cap \beta_{i=1}^{\beta_{1}} U_{\gamma_{t}(p)}(p)$. Then $\mu\left(\mathbb{Q}\left[p, \gamma_{p}\right]\right)>7 / 8$.

If we do this for all $\alpha$ and $p \in C_{\alpha}$, we then have $\mu\left(\mathbb{Q}\left[p, \gamma_{p}\right] \cap \mathbb{Q}\left[q, \gamma_{q}\right]\right)>3 / 4$ for all $p, q$. Suppose $p \in C_{\alpha}, q \in C_{\beta}, \alpha \neq \beta$. Because $\mu\left(\mathscr{B}_{\alpha}-\mathscr{B}_{\beta}\right)=1 / 4$ there exists $f \in \mathbb{Q}\left[p, \gamma_{p}\right] \cap \mathbb{Q}\left[q, \gamma_{q}\right] \cap\left(\mathscr{B}_{\alpha}-\mathscr{B}_{\beta}\right)$. From $f(\alpha)=1, f(\beta)=0$ it follows 
that $p \in U_{A_{j}}, q \in V_{A_{j}}$. And from $f \in \mathbb{Q}\left[p, \gamma_{p}\right]$ it then follows that $U_{\gamma_{p}}(p) \subset U_{A_{j}}$ Similarly, $U_{\gamma_{q}}(q) \subset V_{A_{j}}$. Therefore, $U_{\gamma_{p}}(p) \cap U_{\gamma_{q}}(q)=\varnothing$.

For each $\alpha$, let $U_{\alpha}=\cup\left\{U_{\gamma_{p}}(p) \mid p \in C_{\alpha}\right\}$. Each $U_{\alpha}$ is an open set containing $C_{\alpha}$, and by the preceding paragraph, $U_{\alpha} \cap U_{\beta}=\varnothing$ for $\alpha \neq \beta$. Hence $\left\{C_{\alpha} \mid \alpha<\lambda\right\}$ is well separated.

Incidentally, we have only used the fact that each $p \in C_{\alpha}$ has a local base in $X$ of cardinal $<c$; the points not in $\cup\left\{C_{\alpha} \mid \alpha<\lambda\right\}$ can have any character one pleases. Note also that $X$ is not assumed to satisfy any separation axioms.

It is even possible to relax the condition that $X$ be topological; the same proof yields:

THEOREM 2 [PMEA]. Let $X$ be a closure space [4] such that about each point $x$ of $X$ there is a base of cardinal $<\mathrm{c}$ for the neighborhoods of $x$. Let $\left\{C_{\gamma} \mid \gamma \in \Gamma\right\}$ be $a$ collection of (disjoint) subsets of $X$, such that for every subset $\Lambda$ of $\Gamma$ there are disjoint neighborhoods $N$ and $M$ of $\cup\left\{C_{\gamma} \mid \gamma \in \Lambda\right\}$ and $\cup\left\{C_{\gamma} \mid \gamma \notin \Lambda\right\}$. Then there exist disjoint sets $\left\{N_{\gamma} \mid \gamma \in \Gamma\right\}$ such that $N_{\gamma}$ is a neighborhood of $C_{\gamma}$.

Problem 1. If $N$ and $M$ can always be taken to be open, can the sets $N_{\gamma}$ also be constructed to be open, assuming PMEA?

If the answer is yes, it would imply that every weakly first countable [17], [18] normal space is collectionwise normal under PMEA.

As it is, we have:

THEOREM 3 [PMEA]. Every normal space of character $<\mathrm{c}$ is collectionwise normal.

COROLLARY 1 [PMEA]. Every normal Moore space is metrizable.

In [1], P. S. Alexandroff introduced the concept of a uniform base and showed that a regular Hausdorff space is a metacompact Moore space if, and only if, it has a uniform base. (See also [8], where "uniform" is called "point-regular" and "metacompact" is called "weakly paracompact" or [12], where "metacompact" is called "pointwise paracompact.") We now have:

COROLLARY 2 [PMEA]. Every normal $T_{2}$ space with a uniform base is metrizable.

Another characterization is that of Arhangel'skin [2]: the metacompact Moore spaces are precisely the regular $T_{2}$ images of metric spaces under compact open maps. (A map $f: X \rightarrow Y$ is compact if $f^{-1}\{y\}$ is compact for each $y \in Y$.)

COROLLARY 3 [PMEA]. Metric spaces are preserved under compact open maps if the image space is normal and $T_{2}$.

3. The status of PMEA. The best consistency result on PMEA has been obtained by $\mathrm{K}$. Kunen, who has shown the more general result:

THEOREM 4. If it is consistent that there exist a strongly compact cardinal [7], then there is a model in which the following axiom holds: whenever $(X, \mathcal{S}, \mu)$ is a probability space and $\mathcal{S}$ is a c-complete algebra of subsets of $X$, and $\mu$ is a c-additive 
measure on $\mathcal{S}$, then $\mu$ can be extended to a c-additive measure on $\mathscr{P}(X)$. (This is sometimes called "Fisher's Axiom.")

Kunen constructs his model by starting with one which has a strongly compact cardinal $\kappa$, and "adds $\kappa$ random reals" to the model, so that in the new model $\kappa$ (reinterpreted as an ordinal number-it will no longer be strongly compact!) is the cardinality of the continuum. His proof is a modification of Solovay's in [19]. Kunen also has a shorter and more direct proof that PMEA holds in this model.

On the other hand, R. M. Solovay has shown [19] that the following two axioms are equiconsistent, i.e. if there is a model of one there is a model of the other:

A. ZFC + "there exists a measurable cardinal."

B. $\mathrm{ZFC}+$ " there is a c-additive measure $\mu$ on the real line $\mathbf{R}$ extending the usual Lebesgue measure and defined on all subsets of $\mathbf{R}$."

Now, when $\lambda=\omega_{0}, 2^{\lambda}$ is homeomorphic to the Cantor set, and the product measure can be tied together with the Lebesgue measure on $\mathbf{R}$ by using the Cantor function. So another equivalent condition is

C. ZFC + "PMEA holds for $\lambda=\omega_{0}$."

So the status of PMEA is at least as doubtful as that of measurable cardinals.

4. Other consequences. In survey papers like [13], one can find numerous metrization theorems involving collectionwise normality, a base axiom implying first countability (such as "point-countable base" or " $\delta \theta$-base"), and a generalization of metrizability. We can drop the word "collectionwise" from these theorems under PMEA because of Theorem 3. Actually, most such results follow from Corollary 1 as well, because if we replace "normal" by "regular" we obtain a condition which implies the space is a Moore space. For example, most such metrization theorems in [13] can now be obtained from a recent theorem by Chaber and an older result of Wicke and Worrell.

THEOREM A [5]. Every monotonic $\beta$-space with a $\delta \theta$-base has a base of countable order.

TheOREM B [23]. A regular $T_{2}$ space is a Moore space if, and only if, it is $\theta$-refinable and has a base of countable order.

From another result of Wicke and Worrell, we obtain:

THEOREM 5 [PMEA]. Every normal, $\theta$-refinable space of character $<\mathrm{c}$ is paracompact.

PROof. Every collectionwise normal, $\theta$-refinable space is paracompact [23].

One of the more important generalizations of metric spaces is that of semimetrizable spaces: those admitting a symmetric distance $d$ such that $d(x, y)=0$ if and only if $x=y$, and such that $x$ is in the closure of $A$ if and only if $\inf \{d(x, y) \mid y \in$ $A\}=0$.

THEOREM 6 [PMEA]. Every normal, semimetrizable space is paracompact. 
Proof. A space is a semimetrizable space if, and only if, it is first countable and semistratifiable, and every semistratifiable space is subparacompact [6], hence $\boldsymbol{\theta}$-refinable [23].

In Theorem 6, "paracompact" cannot be improved to "metrizable." For example, the bowtie space in [20] is semimetrizable and paracompact, even Lindelöf, but not metrizable. Of course, the theorem does give us a generalization (under PMEA) of A. H. Stone's classic result that every metrizable space is paracompact.

5. Concluding remarks. All of the theorems and corollaries obtained in this paper from PMEA become false under Martin's Axiom plus the negation of the continuum hypothesis. The metacompact nonmetrizable normal Moore space described in [16, p. 21] that one gets in these models is a counterexample to every one of them, including the metrization theorems one gets by dropping "collectionwise" from the theorems in [13].

The condition "character $<c$ " in Theorem 1 is the best possible, cardinalwise. W. Fleissner has constructed (using no set-theoretic axioms beyond ZFC) a space called "George" which is normal and collectionwise Hausdorff, but not collectionwise normal, and its character is $c$ [16]. However, the following problem also remains open.

Problem 2. Let $X$ be a normal space in which every point has a totally ordered local base. Does PMEA imply that $X$ is collectionwise normal?

\section{REFERENCES}

1. P. S. Alexandroff, On the metrization of topological spaces, Bull. Acad. Polon. Sci. Ser. Math. Astronom. Phys. 8 (1968), 135-140. (Russian)

2. A. V. Arhangel'skii, On mappings of metric spaces, Soviet Math. Dokl. 3 (1962), 953-956.

3. R. H. Bing, Metrization of topological spaces, Canad. J. Math. 3 (1951), 175-196.

4. E. Cech, Topological spaces, Interscience, New York, 1966.

5. J. Chaber, On point-countable collections and monotonic properties, Fund. Math. 94 (1977), 209-219.

6. G. Creede, Concerning semi-stratifiable spaces, Pacific J. Math. 32 (1970), 47-54.

7. F. R. Drake, Set theory, North-Holland, Amsterdam, 1974.

8. R. Engelking, General topology, Polish Scientific Publishers, Warsaw, 1977.

9. W. G. Fleissner, Normal Moore spaces in the constructible universe, Proc. Amer. Math. Soc. 46 (1974), 294-298.

10. __ An introduction to normal Moore spaces in the constructible universe, Topology Proceedings 1 (1976), 47-55.

11. P. R. Halmos, Measure theory, Van Nostrand, Princeton, N. J., 1950.

12. R. W. Heath, Screenability, pointwise paracompactness and metrization of Moore spaces, Canad. J. Math. 16 (1964), 763-770.

13. R. E. Hodel, Some results in metrization theory, 1950-1972, Topology Conference, Lecture Notes in Math., vol. 375, Springer-Verlag, Berlin and New York, 1974, pp. 120-130.

14. F. B. Jones, Concerning normal and completely normal spaces, Bull. Amer. Math. Soc. 43 (1937), 671-677.

15. M. E. Rudin, The metrizability of normal Moore spaces, Studies in Topology, Academic Press, New York, 1975, pp. 507-516.

16. Lectures on set theoretic topology, CBMS Regional Conf. Ser. in Math., no. 23, Amer. Math. Soc., Providence, R. I., 1975.

17. F. Siwiec, On defining a space by a weak base, Pacific J. Math. 52 (1974), 233-245.

18. , Generalizations of the first axiom of countability, Rocky Mountian. J. Math. 5 (1975), $1-60$. 
19. R. M. Solovay, Real-valued measurable cardinals, Axiomatic Set Theory, Proc. Sympos. Pure Math., vol. 13, Amer. Math. Soc., Providence, R. I., 1974, pp. 397-428.

20. L. A. Steen, Conjectures and counterexamples in metrization theory, Amer. Math. Monthly 79 (1972), 113-132.

21. F. D. Tall, A set-theoretic proposition implying the metrizability of normal Moore spaces, Proc. Amer. Math. Soc. 33 (1972), 195-198.

22. __ Set theoretic consistency results and topological theorems concerning the normal Moore space conjecture and related problems, Rozprawy Mat. 148 (1977).

23. J. M. Worrell and H. H. Wicke, Characterizations of developable topological spaces, Canad. J. Math. 17 (1965), 820-830.

Department of Mathematics, Auburn University, Auburn, Alabama 36830

Current address: Department of Mathematics, Computer Science and Statistics, University of South Carolina, Columbia, South Carolina 29208 